Objectives: We aimed to apply an unbiased single cell approach to assess protein expression levels of phenotypic and functional markers within the human $\mathrm{CD}^{+} \mathrm{T}$ cell compartment in subjects with prototypical BD in order to identify cell populations of potential biologic relevance.

Methods: We determined single cell surface/intra nuclear expression levels of CD3, CD4, CD8, CD127, CD25, CD45RA, CCR7, FoxP3, HELIOS, Ki67, HLA$\mathrm{DR}, \mathrm{CD} 38, \mathrm{CD} 39$ in PBMC by flow cytometry and computed the representation of all mathematically possible cell populations within pre-defined starting populations. PBMC from BD subjects $(n=13)$, healthy donors $(H D)(n=25)$ and diseased subjects with non-BD auto-immune uveitis $(n=11$; VKH, Sarcoidosis, and HLAB27 associated uveitis) were used. BD subjects met ISG criteria and were Arab or Chinese. $62 \%$ had pan-uveitis, $23 \%$ major vascular disease, and $7 \%$ parenchymal CNS disease. $46 \%$ were HLA-B51 carriers.

Results: Computation of all populations defined by CD127, CD25, CD45RA, CCR7, FoxP3, HELIOS, Ki67, HLA-DR within the CD4 ${ }^{+} \mathrm{T}$ cell compartment yielded a total of $6560\left(3^{\wedge} 8-1\right)$ cell populations out of which 45 reached a significance level of $p \leq 0.000001$ in ANOVA testing to differentiate 3 groups (BD, non$\mathrm{BD}$ uveitis, and HD). All of these populations comprised sub-types of the human regulatory $\mathrm{T}$ (Treg) cell compartment with a strong predominance of non-proliferating, non-activated, FoxP3 ${ }^{+} \mathrm{HELIOS}^{+}$Treg carrying central-memory phenotypes $\left(\mathrm{CD}_{45 \mathrm{RA}}^{-}, \mathrm{CCR}^{+}\right)$. Unpaired testing of $\mathrm{BD}$ vs non-BD revealed 43 distinct cell populations at a significance level of $\mathrm{p} \leq 0.002$ representing $C D 25^{+}$non-Treg; comparison of BD vs HD uncovered 58 populations at a significance level of $p \leq 0.0001$ representing FoxP3 ${ }^{+} \mathrm{HELIOS}^{+}$subpopulations, and non-BD vs $\mathrm{HD}$ identified 61 populations at $\mathrm{p} \leq 0.001$, comprising $\mathrm{CD} 25^{+} \mathrm{CD} 127^{ \pm} \mathrm{FoxP}^{ \pm}$, but consistently HELIOS ${ }^{-}$cells. A separate analysis using CD38, CD39, CD227, TIGIT, CD45RA, CCR7 within the $\mathrm{CD} 3^{+} \mathrm{CD} 4^{+} \mathrm{CD} 8^{-} \mathrm{CD} 127^{-} \mathrm{CD} 25^{+}$compartment that contains most human Treg, showed 48 populations $(\mathrm{p} \leq 0.0001)$ in 3 -way comparison pointing to high significance of TIGIT and CD226 staining Treg subpopulations, and $18 \mathrm{popu}-$ lations with differential expression of $C D 39^{+}$between $B D$ and non-BD diseased subjects. TIGIT and CD226 co-expressing Treg $\left(\mathrm{CD}^{2} 127^{-} \mathrm{CD} 25^{+}\right)$subpopulations also reached significance $(p \leq 0.02)$ in a longitudinal paired analysis of 7 BD subjects in active vs inactive disease states, as did 56 out of 6560 populations within total $\mathrm{CD} 4^{+} \mathrm{T}$ cells, mostly representing non-Treg cells in active $\mathrm{BD}$.

Conclusions: Differential expression of $\mathrm{CD}^{+}$Treg and non-Treg cells shapes the immune-phenotype of BD vs states of health and non-BD autoimmune diseases that have phenotypic overlap with BD (uveitis). The populations with the highest significance for differentiating BD from healthy states seem to exist within the HELIOS ${ }^{+} \mathrm{FOXP3}^{+}$compartment of non-activated, non-proliferating Treg, suggesting relevance of a true Treg phenoptype. This is likely not specific to BD. NonTreg $\mathrm{CD}_{25}{ }^{+}$cell populations seem more indicative of BD vs non-BD uveitic disease as well as of clinically active BD while populations with high CD39 expression may signify non-BD states of immune-mediated disease.

Disclosure of Interest: None declared

DOI: 10.1136/annrheumdis-2018-eular.7160

\section{AB0032 B-CELL SUBPOPULATIONS IN NEWLY DIAGNOSED EORA AND YORA PATIENTS}

K. Thorarinsdottir, A. Camponeschi, L. Jacobsson, I.-L. Mårtensson, I. Gjertsson. Department of Rheumatology and Inflammation Research, University of Gothenburg, Gothenburg, Sweden

Background: B-cells are thought to have an important role in rheumatoid arthritis (RA). This is demonstrated by the success of B-cell depleting therapy as well as the negative prognostic value of anti-citrullinated protein antibodies (ACPA) ${ }^{1}$. However, the pathogenesis of the disease is unclear. Studies have suggested that there are differences in disease characteristics between elderly-onset RA patients (EORA, defined by disease onset at $\geq 60$ years of age) and youngeronset RA patients (YORA, with disease onset $<60$ years of age). ${ }^{2}$

Objectives: Our aim was to study the B-cell subpopulations in newly diagnosed EORA and YORA patients. We investigated whether there were differences in Bcell subpopulations between the groups and whether there was a correlation between B-cell subpopulations and disease activity, autoantibody profile and inflammatory parameters in these two RA patient groups.

Methods: Treatment-naive EORA $(n=29)$ and Yora $(n=31)$ patients with newly diagnosed RA were included at their first visit to the Rheumatology clinic. The patients' clinical response (DAS28), autoantibodies and B-cells were assessed. Flow cytometry was used for the analysis of cellular surface markers on leukocytes in peripheral blood: CD19, CD27, CD24, CD27, CD38, PD-1, PD-L1, IgG, $\lg \mathrm{D}$ and $\lg \mathrm{M}$. Non-parametric tests were used for comparing groups and Spearman's test was used for correlation.

Results: We found a correlation between the ACPA titers and the frequency of the $\mathrm{CD}^{2} 7^{+}$and $\mathrm{CD} 27^{-}$memory $\mathrm{B}$ cell populations in EORA patients but not in YORA patients. This was further supported by a correlation of the ACPA titer and $\mathrm{IgG}^{+} \mathrm{B}$ cells in the EORA patients $(\mathrm{r}=0.7, \mathrm{p}=0.003)$ and not in the YORA patients. There was neither a correlation between age and ACPA titer nor between age and memory $B$ cell populations. We did not find any significant difference between the B cell subpopulations in the two patient groups.

Conclusions: Our results suggest that the memory B cell compartment in peripheral blood in EORA patients reflects the ACPA titer. This was not seen in the YORA patients. The mechanisms behind these findings need to be further elucidated.

\section{REFERENCES :}

[1] Bugatti S, Vitolo B, Caporali R, Montecucco C, Manzo A. B cells in rheumatoid arthritis: from pathogenic players to disease biomarkers. Biomed Res Int 2014;2014:681678.

[2] Kobak S, Bes C. An autumn tale: geriatric rheumatoid arthritis. Ther Adv Musculoskelet Dis 2018;10:3-11.

Disclosure of Interest: None declared

DOI: 10.1136/annrheumdis-2018-eular.6459

\section{AB0033 B REGULATORY CELLS POSITIVELY CORRELATE WITH ANTIBODIES AGAINST SS-A RO52 IN SYSTEMIC SCLEROSIS}

A. Mavropoulos, A. Gkoutzourelas, T. Simopoulou, C. Liaskos, D.P. Bogdanos, L. I. Sakkas. Rheumatology and Clinical Immunology, Faculty of Medicine, School of Health Sciences, University of Thessaly, Larissa, Greece

Background: We and Japanese Investigators have shown that IL-10-producing regulatory $B$ cells (B10 cells) are decreased in systemic sclerosis (SSc). ${ }^{12}$ Contrary to the Japanese, we did not find a negative correlation between B10 cells and SSc-specific autoantibodies (autoabs) against centromere, Scl-70 or RNA polymerase III. $^{3}$ Since we found anti-Ro52 SS-A antibodies in approx. 30\% of patients with $\mathrm{SSc},{ }^{4}$ this being the $3 \mathrm{rd}$ most frequent autoantibody in this disease, we considered what is the relation of $\mathrm{B} 10$ cells with anti-Ro52 antibodies.

Objectives: We examined the number and function of Bregs in SSc in relation to anti-Ro52 autoabs.

Methods: Serum samples and PBMCs were collected from 40 SSc patients (15 anti-Scl-70, 20 anti-CEN and 5 anti-RNA pol III) and were further divided according to anti-Ro52-positivity into 22 anti-Ro52(+) and 18 anti-Ro52 (-). All serum samples were tested for the presence of disease-specific autoantibodies against Scl-70, CENP, RNA-pol, and against Ro52 using a line assay (Euroimmun Germany). The function of Bregs was determined by the ability to express IL-10 following activation with ODN 2006 (TLR-9). Percentages of transitional (CD19 + CD24highCD38high) and memory (CD19 +CD27+CD24 high) Bregs were assessed by flow cytometry using fluorochrome conjugated antibodies (BD Biosciences)

Results: IL-10(+) Bregs (B10) were significantly elevated in SSc patients $(6.7 \%$ $\pm 2.6 \% \mathrm{n}=15$ ) with high-titre antibodies against Ro52 (mean anti-Ro52 arbitrary units $A U>100$; positivity cut-off $A U>20)$ compared to patients $(4.2 \% \pm 1.9 \%, n=22$ totally negative for Ro52 (mean AU <10) $(p=0.03)$. Transitional Bregs were also significantly increased in all SSc patients tested positive for anti-Ro52 autoantibodies $(7.5 \% \pm 1.9 \%)$ compared to SSc patients negative for anti-Ro52 autoantibodies $(3.7 \pm 0.8, p=0.02)$. Furthermore, transitional Bregs positively correlated with anti-Ro52 antibody levels $\left(r^{2}=0.39 p=0.01\right)$. In contrast, memory Bregs were not significantly different between anti-Ro52-positive $(14.1 \% \pm 2.7 \%)$ and -negative SSc patients $(11.8 \% \pm 2.2 \%)(p>0.05)$.

Conclusions: IL10-producing Bregs are higher in SSc patients with high antiRo52 antibodies and transitional Bregs correlated with antiRo52 antibodies in patients with SSc suggesting that this autoantibody could be a potential marker of Breg efficiency.

\section{REFERENCES}

[1] Mavropoulos A, et al. Arthritis Rheumatol 2016;68(2):494-504.

[2] Matsushita T, et al. Rheumatology 2016;55(2):263-67.

[3] Mavropoulos A, et al. Clin Immunol 2017;184:26-32.

[4] Liaskos C, et al. Autoimmunity 2017;50(7):414-421. 
Disclosure of Interest: None declared DOI: 10.1136/annrheumdis-2018-eular.7186

\section{AB0034 LOKING FOR A SLE SIGNATURE ON PERIPHERAL B CELL SUBSETS: DOES A PREPONDERANT CD38 +PLASMABLAST-SUBPOPULATION LACK CD73 AS A SIGN OF A DISTURBED ADENOSINE AXIS?}

M. Siekierka-Harreis, M. Schroedter, G. Chehab, J. Richter, M. Schneider, G. Pongratz. Rheumatology, Heinrich-Heine University Duesseldorf, Medical Faculty, Duesseldorf, Germany

Background: Systemic Lupus Erythematosus (SLE) is an autoimmune disorder characterised by polyclonal Bcell activation, production of dsDNA-autoantibodies and cytokines. Subsets of Bcells play a central role in SLE-pathogenesis. The inflammatory milieu is characterised by the accumulation of adenosine, which confers immunosuppressive effects.

Objectives: In SLE, the role of CD73, an enzyme involved in the extracellular generation of adenosine from ATP, is not well characterised. This study aimed to characterise expression of CD73 Bcell subsets of SLE-patients as compared to healthy controls $(\mathrm{HC})$.

Methods: Bcell subsets were characterised from peripheral blood of 23 SLE patients attending the outpatient clinic at the Rheumatology Unit of University Hospital Düsseldorf and of $15 \mathrm{HC}$ by FACS. All patients fulfilled the revised SLEcriteria of ACR and were randomly collected in clinical remission state (SLEDAI $1.1 \pm 1.9)$.

Results: By comparison of Bcell subsets between SLE and HC, CD38 was dominantly expressed by SLE-patients (SLE $74.2 \% \pm 12.9 \%$ vs. HC $64.2 \%$ $\pm 12.2 \%$; $p(M W U)=0.018)$. Furthermore, SLE-patients showed an increase in CD19 + lgD CD27+CD38 high plasmablasts (SLE 2.1\% $\$ 3.4 \%$ vs $\mathrm{HC} 0,4 \%$ $\pm 0.4 \%, \mathrm{p}(\mathrm{MWU})<0.001)$. Furthermore, SLE-plasmablasts showed decreased CD73 expression as compared to HC(SLE $2.1 \% \pm 1.9 \%$ vs HC $3.5 \% \pm 2.2 \%$; p $(\mathrm{MWU})=0.034)$. SLE-Bcells revealed a trend towards an augmented CD38highCD138+plasmacell fraction (SLE $0.40 \% \pm 0.5 \%$ vs HC $0.08 \%$ $\pm 0.07 \%$; $p=0.07$ ), without any difference in CD73 expression. On the other hand, exhausted-memory B cell fraction (CD19 + IgD CD27-CD21-CD138-), showed an increased CD73 expression in SLE (SLE 13.7\% $\pm 9.2 \%$ vs HC $6.2 \%$ $\pm 5.4 \% ; p=0.004)$.

Conclusions: Our study confirms CD38 +plasmablasts as being increased in peripheral blood from SLE patients as compared to HC. Furthermore, the data reveal a deficiency for CD73 on SLE plasmablasts, which suggests a decreased anti-inflammatory capacity of SLE plasmablasts as compared to $\mathrm{HC}$, supporting the notion of a disturbed adenosine axis in SLE. On the other hand, the enlarged CD73 +exhausted memory pool in SLE could point to an accelerated flow of CD73 +B cells into an exhausted B cell fraction. These findings support the hypothesis of dysregulation of the adenosine axis in SLE even in inactive SLE patients.

\section{REFERENCES:}

[1] Szabo K, Papp G, Szanto A, Tarr T, Zeher M. A comprehensive investigation on the distribution of circulating follicular $T$ helper cells and $B$ cell subsets in primary Sjogren's syndrome and systemic lupus erythematosus. Clinical and experimental immunology 2016;183(1):76-89.

[2] Jacobi AM, Reiter K, Mackay M, Aranow C, Hiepe F, Radbruch A, et al. Activated memory $B$ cell subsets correlate with disease activity in systemic lupus erythematosus: delineation by expression of CD27, IgD, and CD95. Arthritis and Rheumatism 2008:58(6):1762-73.

[3] Deaglio S, Dwyer KM, Gao W, Friedman D, Usheva A, Erat A, et al. Adenosine generation catalyzed by CD39 and CD73 expressed on regulatory $T$ cells mediates immune suppression. The Journal of Experimenta Medicine 2007;204(6):1257-65.

[4] Hasko G, Linden J, Cronstein B, Pacher P. Adenosine receptors: therapeutic aspects for inflammatory and immune diseases. Nature Reviews Drug Discovery 2008;7(9):759-70.

Disclosure of Interest: None declared DOI: 10.1136/annrheumdis-2018-eular.4451
$\mathrm{AB} 0035$

A PHYSIOLOGICAL NETWORK OF IGG AUTOANTIBODIES TARGETING G PROTEIN COUPLED RECEPTORS

O.C. Marques $^{1}$, A. Marques ${ }^{1}$, R. de Vito ${ }^{2}$, L.M. Giil ${ }^{3}$, J. Rademacher ${ }^{4,5}$, J. Günther ${ }^{4,5}$, G. Marschner ${ }^{1}$, S. Pitann ${ }^{1}$, S. Adler ${ }^{6}$, D. Dragun ${ }^{7}$, P. Lamprecht ${ }^{1}$ A. Müller ${ }^{1}$, X. Yu ${ }^{8}$, F. Petersen ${ }^{8}$, H. Heidecke ${ }^{9}$, B.E. Engelhardt ${ }^{2}$, G. Riemekasten ${ }^{1}$ ${ }^{1}$ Reumathology, University of Lübeck, 23562 Lübeck, Germany; ${ }^{2}$ Computer Science, University of Princeton, Princeton, USA; ${ }^{3}$ Deaconess Hospital, University of Bergen, Bergen, Norway, ${ }^{4}$ Reumathology, Charité University Hospital; ${ }^{5}$ Cell Autoimmunity Group, German Rheumatism Research Center, Berlin, Germany; ${ }^{6}$ Reumathology, University Hospital Bern, Bern, Switzerland; ${ }^{7}$ Dept. of Nephrology and Cardiovascular Research, Campus Virchow, Charité University Hospital, Berlin; ${ }^{8}$ Research Center Borstel, Airway Research Center North (ARCN), Members of the German Center for Lung Research (DZL), Borstel; ${ }^{9}$ CellTrend $\mathrm{GmbH}$, Luckenwalde, Germany

Background: Since the time when Paul Ehrlich conceived the term "horror autotoxicus", autoantibodies have been associated with the development of autoimmune diseases. However, several works have recently shown the presence of autoantibodies in sera from healthy subjects $(n=489)$, who do not develop autoimmune diseases.

Objectives: Here, we report a network of immunoglobulin $\mathrm{G}(\mathrm{lgG})$ autoantibodies targeting G protein-coupled receptors (GPCRs), growth factors and growth factorrelated molecules in sera from healthy subjects.

Methods: Autoantibody levels in sera were assessed using ELISA. Autoantibody network was analysed by exploratory factor analysis (EFA), dandelion plo method, hierarchical clustering, and multi-study factor analysis (MFA). We also reverse engineered autoantibody functions through in silico evaluation of autoantibody target interactions using STRING and gene ontology (GO). To test the autoantibodies functionality we assessed the in vitro production of IL- 8 by PBMCs and neutrophil migration in response to $\mathrm{IgG}$ from healthy subjects as well as ETAR immunised and control mice. Leukocyte cellularity to secondary immune organs was analysed comparing ETAR-immunised with control mice.

Results: Gender, age and the presence of pathological conditions (systemic sclerosis $n=84$, Alzheimer's disease $n=91$ and ovarian cancer $n=207$ ) changed corre lations between the autoantibodies and their hierarchical clustering distribution. Notably, subjects at age below and above 65 years or with pathological conditions exhibited particular autoantibody hierarchical clustering signatures. In addition, females at age above 65 years, representing the group of subjects with higher risk to develop SSc, displayed the closest link to SSc in terms of autoantibody hierarchical clustering. Finally, autoantibody directed against the endothelin receptor type A (ETAR) showed an essential role in the autoantibody network by orches trating neutrophil trafficking in vitro and in ETAR-immunised mice.

Conclusions: Our data provide a framework for the existence of a physiological network of autoantibodies and reveal a new paradigmatic view on these physiological molecules.

Disclosure of Interest: None declared

DOI: 10.1136/annrheumdis-2018-eular.4461

\section{$\mathrm{AB} 0036$ \\ ROLE OF PROGRAMMED DEATH-1 PATHWAY ON CD8+ T CELLS CYTOTOXICITY IN PRIMARY BILIARY CHOLANGITIS}

S. Zhang, X. Tao, L. Wang, L. Zhao, J. Sun, Z. Chen, H. Chen, F. Zhang. Peking Union Medical College Hospital, Beijing, China

Background: Primary Biliary Cholangitis (PBC) is a chronic progressive autoim mune disease. It has been proven that there was abnormal activation of CD $8+T$ cells. Previous studies have shown that abnormal expression programmed death 1 (PD-1) and its ligand (PD-L1) in PBC. However, no study was found to confirm the abnormality of PD-1/PD-L1 pathway in CTL in PBC.

Objectives: To investigate the role of PD-1 and its ligand PD-L1 on CD8 ${ }^{+} T$ cells cytotoxicity in the immunological mechanism of PBC

Methods: The expression of PD-1 in peripheral CD8 ${ }^{+} \mathrm{T}$ cells of 69 patients diagnosed with $\mathrm{PBC}$ as well as 58 health controls $(\mathrm{HC})$ was detected by flow cytometry. Plasma cytokines related to PD-1/PD-L1 pathway were detected by ELISA. The co-localization of PD-1 and CD-8, PD-L1 and CK19 in portal region in liver biopsy was analysed by immunofluorescence assay. Meanwhile, the relative gene expression of PD-1 regulatory pathway in $\mathrm{CD}^{+} \mathrm{T}$ lymphocytes were meas ured by RT-PCR. And co-culture of PD- $1^{ \pm} \mathrm{CD} 8^{+} \mathrm{CTL}$ and HiBEC was done in vitro to detect the cytotoxicity, proliferation and cytokine expression of $C D 8^{+} \mathrm{CTL}$ and the apoptosis of HiBEC.

Results: The proportion of peripheral PD- $1^{+} \mathrm{CD} 8^{+} \mathrm{T}$ cell decreased in PBC $(12.0 \% \pm 8.8 \%)$ than $\mathrm{HC}(19.9 \% \pm 12.5 \%)(\mathrm{p}<0.001)$. The plasma concentration of $\mathrm{IL}-10, \mathrm{IFN}-\gamma$ and TGF- $\beta$ in the PBC group were higher than that in $\mathrm{HC}(8.29$ $\pm 9.00 \mathrm{pg} / \mathrm{ml}$ v.s. $4.43 \pm 5.08 \mathrm{pg} / \mathrm{ml}, \mathrm{p}=0.0066 ; 51.94 \pm 52.94$ vs $26.71 \pm 26.26 \mathrm{pg} / \mathrm{ml}$, $\mathrm{p}=0.0015 ; 1302.0 \pm 1972.8 \mathrm{pg} / \mathrm{ml}$ vs $205.8 \pm 298.9 \mathrm{pg} / \mathrm{ml}, \mathrm{p}=0.0018$, resp.). Compared with $\mathrm{HC}(\mathrm{n}=19)$, Tbet gene expression in $\mathrm{CD}^{+}$T lymphocyteswas increase 